\title{
Comparative Study of Mechanisms for Web Service Discovery based on Centralized Approach Focusing on UDDI
}

\author{
Netra Patil \\ Associate Professor \\ Sinhgad Institute of Business \\ Administration and Research, Pune
}

\author{
Dr. Arpita Gopal \\ Director MCA \\ Sinhgad Institute of Business Administration \\ and Research, Pune
}

\begin{abstract}
A Web service is a set of related application methods that can be accessed programmatically over the Internet. Businesses can dynamically mix and match Web services to perform complex functionality with minimal programming. Web services allow buyers and sellers all over the world to find each other, connect dynamically, and execute transactions in real time with minimal human interaction. Web services are self-contained, self-describing modular applications that can be published, located, and invoked across the Web. While creating web applications using web service technology there is a need for selecting new web services which best suit our need at the moment. For doing this we require to specify our criteria for selection of web service. Apart from this, appropriateness and optimality are the challenging issues at the time of web service consumption. Web services are important for interoperability of applications and integration of large scale distributed systems irrespective of platform. Web service discovery mechanisms are equally important, especially as the latter has turn to be difficult task. This paper reviews the latest mechanism, techniques, architectures and models that have evolved in the web service discovery area.
\end{abstract}

\section{Keywords}

UDDI, P2P, METEOR-S, QoS, WSMX

\section{INTRODUCTION}

There is increasing demand for dynamically integrating different business applications and services. Several architectures, techniques and mechanisms have been suggested for more flexible and scalable process environments. The growth of web services and service oriented architecture offer an attractive basis for realizing such architectures and mechanisms.

Web Services have marked current web engineering methodologies and are universally supported by IT vendors and users. In short they are interoperable software components that can be used in application integration and component based application development. As the demand for web service consumption is increasing, a series of questions concerning the methods and procedures to discover the most suitable web service arises. In fact there is much hiding behind the discovery of a web service. This work aims to examine and analyze the different proposals in the area.

Discovery of Web services is of great interest and is a fundamental area of research in distributed computing. Many researchers have focused on discovering web services through a centralized UDDI registry [18, 19, 26]. Although centralized registries can provide effective methods for the discovery of Web services, they suffer from problems associated with having centralized systems such as a single point of failure, and bottlenecks. In addition, other issues relating to the scalability of data replication, providing notifications to all subscribers when performing any system upgrades, and handling versioning of services from the same provider have driven researchers to find other alternatives.

As Web services have begun to expand across the internet, users need to be able to efficiently access and share Web services. Production and interoperability of larger number of web services have lead to the emergence of new standards on how services can be published, discovered or used. Hence, mechanisms are required for efficient selection of appropriate web service instance in terms of quality and performance factors at the moment of the Web Service consumption.

The discovery mechanism should offer a number of capabilities, recognizable at both development and execution time. During development, one may search a web service repository for information about available services. At execution, client applications may use this repository to discover all instances of a web service that match a given interface.

Web service discovery mechanism in general is the task of locating a machine-processable description of a web service that may have been previously unknown and that meets certain functional criteria.

\section{APPROACHES FOR WEB SERVICE DISCOVERY}

Traditionally, the Web service discovery processes involved manual interference. A set of Web service descriptions is discovered according to user requirements. These service descriptions are manually scanned and those services which satisfy user requirements are selected and composed. In distributed system integration environment, such manual intervention is impractical, cumbersome and time consuming. The approaches to Web services discovery can be classified as centralized and decentralized. UDDI falls under fully centralized approach that supports replication where central registries are used to store Web service descriptions. Having realized that replicating the UDDI data is not a scalable approach several decentralized approaches have been proposed. Two major operators, namely IBM and Microsoft provide public UDDI service.

Web service discovery mechanisms include a series of registries, indexes, catalogues, agent based and Peer to Peer- 
P2P solutions. The most dominating among them is the Universal Description Discovery and Integration-UDDI standard that is currently in version 3 .

\section{RELATED WORK}

\subsection{Mechanisms for Web Service Discovery based on Decentralized Approach :}

Bernstein, Abraham, and Mark Klein [1] described a new service retrieval approach based on the sophisticated use of process ontologies. This approach offers qualitatively higher retrieval precision than existing (keyword and table based) approaches without sacrificing recall and computational tractability/scalability.

I. Toma, Brahmananda Sapkota, James Scicluna, Juan Miguel Gomez, Dumitru Roman, and Dieter Fensel [2,11] addressed the discovery problem for a specific Semantic Web service execution environment, namely Web Service Modeling Execution Environment (WSMX). More precisely, they proposed a P2P Discovery mechanism for Semantic Web services descriptions that are registered with Web Service Modelling Execution Environment.

D. Martin, M. Paolucci, S. Mcllraith, M. Burstein, D. McDermott, D. McGunneess, B. Barsia, T. Payne, M. Sabou, M. Solanki, N. Srinivasan, and K. Sycara [3] and D. Roman, H. Lausen, and U.Keller [4] are attempting to provide a formal way of expressing service provider's capabilities and user's requirements. These initiatives are mainly focused on knowledge representation aspects. Apart from knowledge representation, the web service discovery is a complex task and need to consider the context of its availability and usability.

Eyhab Al-Masri and Qusay H. Mahmoud [5] proposed a solution by introducing the Web Service Relevancy Function (WsRF) used for measuring the relevancy ranking of a particular Web service based on QoS metrics and client preferences for the purpose of finding the best available Web service during Web services' discovery process based on a set of given client QoS preferences.

F. Banaei-Kashani, C.-C. Chen, and C. Shahabi [8] and I. Toma, B. Sapkota, J. Secuila, J. M. Gomez, D. Roman, and C. Bussler [11] attempted to bring web service discovery mechanism on top of Peer-to-Peer network thereby reducing human intervention which is concerned with resource linking but nothing has been mentioned about the applications that process these resources.

Fatih Emekci, Ozgur D. Sahin, Divyakant Agrawal, Amr El Abbadi [9] proposed a structured peer-to-peer framework for web service discovery in which web services are located based on both service functionality and process behavior. In addition, they integrate a scalable reputation model in this distributed peer-to-peer framework to rank web services based on both trust and service quality.

Gang Zhou, Jianjun Yu, Rui Chen, Hui Zhang [10] proposed a peer-to-peer framework, which implements an enhanced Skip Graph named ServiceIndex as the overlay network for service discovery. To guarantee discovery efficiency, ServiceIndex schemed WSDL-S (Web Services Semantics) as Semantic Web Services description language and extracted its semantic attributes as indexing keys in Skip Graph.

Jacek Kopeck'y [12] intended to research an approach to Semantic Web Service discovery that splits the task of finding the most appropriate offer from available web services into static discovery and then dynamic offer discovery. Initially it will find all available web services fulfilling the user's goal, by interacting with discovered services, find offers relevant for the goal, filter out the offers which do not meet the user's constraints, rank the offers according to user's preferences and choose one to be invoked and then use the selected service.

Jorge Cardoso and Amit Sheth [15] presented a methodology and a set of algorithms for Web service discovery based on three dimensions: syntax, operational metrics, and semantics. In this approach, Web service discovery is not only based on functional requirements, but also on operational metrics. Operational metrics of web services are described by QoS model representing quantitative and qualitative characteristics of an e-workflow application necessary to achieve initial requirements.

K. Verma, K. Sivashanmugam, A. Sheth, A. Patil, S. Oundhakar and J. Miller [17] presented METEOR-S Web Services Discovery Infrastructure (MWSDI), a scalable infrastructure for semantic publication and discovery of Web services. They presented two algorithms for semantic publication and discovery using WSDL descriptions.

Mario Schlosser, Michael Sintek, Stefan Decker, Wolfgang Nejdl [20] proposed a graph topology which allows very efficient broadcast and search, and provide an efficient topology construction and maintenance algorithm which, vital to symmetric peer-to-peer networks, neither requires a central server nor super nodes in the network.

Patrick C. K. Hung And Haifei Li [21] proposed a token based approach for web services discovery based on the tradeoff between Quality and Cost of Service (QoS and CoS) to quantify the QoS and CoS for achieving integrative solutions. One of the negotiation strategies for achieving integrative solutions for both parties is called logrolling. Logrolling is an important step in web service discovery process in which both web services providers and web services requestors can find appropriate partners.

Qiang He, Jun Yan, Yun Yang, Ryszard Kowalczyk, Hai Jin [23] proposed a peer-to-peer based decentralized service discovery approach named Chord4S. To improve data availability, Chord4S distributes the descriptions of functionally-equivalent services over the virtual segment of the circle. An efficient routing algorithm is provided to facilitate queries of multiple candidate service providers.

Shou-jian Yu, Xiao-kun Ge, Jing-zhou Zhang, Guo-wen Wu [24] presented a flexible Web service discovery architecture by combining semantic Web service with P2P networks. This system does not need a central registry for Web service discovery. An ontology-based approach to capture real world knowledge for semantic service details is used. They implement distributed hash table (DHT) based catalog system in P2P system to index the ontology information and store the index at each peer. DHT supports only exact match lookups, so they have also improved matching algorithms for flexible service discovery.

Shuiguang Deng, Zhaohui Wu, Jian Wu and Ying Li [25] are proposing a two-phase semantic-based service discovery mechanism to discover services in precise, efficient and automatic way. Comparing with other approaches, this mechanism has two main characteristics like it takes into account the interface dependencies implied within an operation while performing matchmaking and it supports twolevel matchmaking, namely operation matchmaking and operation-composition matchmaking.

U. Keller, R. Lara, A. Polelres, I. Toma, M. Kifer, and D. Fensel [27] described different levels of service matches. It is understood that service matches are mandatory but not sufficient for Web service discovery. 


\subsection{Mechanisms for Web Service Discovery based on Centralized Approach :}

Eyhab Al-Masri and Qusay H. Mahmoud [6] provide qualitydriven centralized discovery approach using Web service broker (WSB). In this model, service providers publish service information in the UDDI or search engines. WSB performs the tasks of gathering web services spread over the web and monitoring their behavior based on various QWS metrics automatically without needing any human intervention. Its interface allows clients to express appropriate service queries based on QWS. As clients receive response, they can invoke services.

Eyhab Al-Masri and Qusay H. Mahmoud [7] propose Web Service Crawler Engine (WSCE), a crawler that can capture service information from various accessible resources over the Web like UBR, search engines and service portals, to help in searching of Web services on the Web.

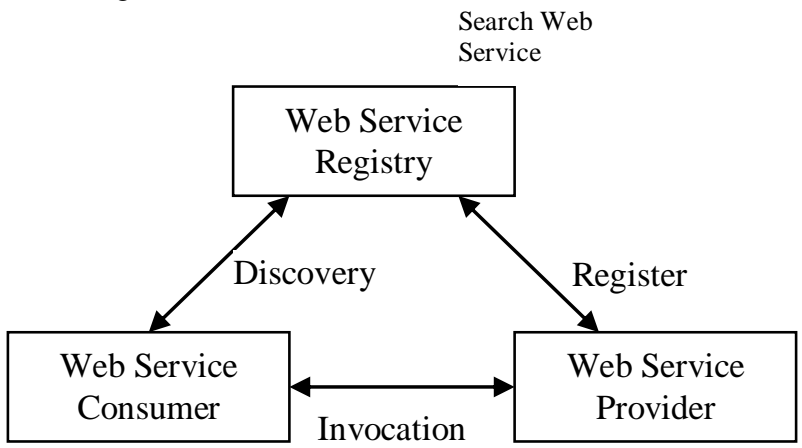

Fig 1: Architecture for Web Service Discovery based on Centralized Approach

K. Sivashanmugam, K. Verma, and A. Sheth [16] proposed METEOR-S Web Service Discovery Infrastructure(MWSDI), an ontology based infrastructure to provide access to private and public registries divided based on business domains and grouped into federations for enhancing the discovery process. METEOR-S provides a discovery mechanism for publishing Web services over a federated registry sources but, similar to the centralized registry environment, it does not provide any means for advanced search techniques which are essential for locating appropriate business applications. In addition, having a federated registry environment can potentially provide inconsistent policies to be employed which will significantly have an impact on the practicability of conducting inquiries across the federated environment and can at the same time significantly affect the productiveness of discovering Web services in a real-time manner across multiple registries.

M. Paolucci, T. Kawamura, T. Payne, and K. Sycara [18] focused on discovering Web services through a centralized UDDI registry. Centralized registries can provide effective methods for the Web service discovery, but they suffer from problems associated with having centralized systems such as a single point of failure, and delayed delivery of notifying updated service description. Other issues relating to the scalability of data replication, and handling versioning of services from the same provider are driving researchers to find other alternatives.

\section{METHODOLOGY OF RESEARCH}

The methodology of our research is described as follows : Conduct literature review on web service discovery mechanisms. We will build a database of the accessible Web service resources including UDDI Business Registries, service directories/portals, and search engines indices. Also collect Web service information including metadata and WSDL documents. Verify and validate the number of accessible Web services. Analyze retrieved Web services by obtaining service metadata, WSDL documents, supported protocols, serviceability, types of technologies used. Build a set of available resources that provide a list of Web services that can later be used as the input to our experiments.

Dimensions of evaluation of web service discovery mechanism are as follows -

The efficiency of the approach will be evaluated in two dimensions: volume and complexity of the necessary semantic description, and the performance and scalability of the discovery process. Testing of the proposed new discovery mechanism will be by developing two interoperating applications trying to consume the appropriate web service among various available web services.

\section{CONCLUSIONS}

Web services can be located through a public business registry, a private business registry, or a WSIL document. UDDI manages the discovery of Web services by relying on a distributed registry of businesses and their service descriptions implemented in a common XML format.

The web service discovery mechanisms discussed above try to achieve objective to enhance efficiency in the matching and binding appropriate web service.

The work on discovery mechanisms tries to obtain resulting mechanism not only applicable to web services, but webbased or other software components in general. This may require introducing some additional specifications about the platform, the system requirements etc. Many approaches have been discussed using different view-points. From starting the centralized approach, UDDI based mechanisms are very optimistic. Web service discovery mechanisms are more important than web searching, because they facilitate the need for collaboration among business applications and consumers over widely accepted web standards.

In this paper, several approaches mainly including centralized approach UDDI, have been discussed using different viewpoints. Web service Discovery mechanisms have a role even more important than Web searching, because they facilitate the need for collaboration among business applications and consumers.

\section{REFERENCES}

[1] Bernstein, Abraham, and Mark Klein [2002]: Discovering Services: Towards High Precision Service Retrieval. Proceedings of the CaiSE workshop on Web Services, e- Business, and the Semantic Web: Foundations, Models, Architecture, Engineering and Applications. Toronto, Canada

[2] Brahmananda Sapkota, Dumitru Roman, Dieter Fensel [2005]: Distributed Web Service Discovery Architecture.

[3] D. Martin, M. Paolucci, S. Mcllraith, M. Burstein, D. McDermott, D. McGunneess, B. Barsia, T. Payne, M. Sabou, M. Solanki, N. Srinivasan, and K. Sycara [2004]: Bringing Semantics to Web Services: The OWL-S Approach. In Proceedings of the First International Workshop on Semantic Web Services and Web Process Composition.

[4] D. Roman, H. Lausen, and U.Keller [2004]: Web Service Modelling Ontoloty - Standard. WSMO Working Draft, v0.2.

[5] Eyhab Al-Masri and Qusay H. Mahmoud [2007]: Discovering the Best Web Service. WWW 2007. 
[6] Eyhab Al-Masri, Qusay H. Mahmoud [2008]: Investigating Web Services on the World Wide Web. WWW 2008 Track: Web Engineering - Web Service Deployment. [7] Eyhab Al-Masri, Qusay H. Mahmoud [2008]: Toward Quality- Driven Web Service Discovery. ITPro May 2008 IEEE Computer Society.

[8] F. Banaei-Kashani, C.-C. Chen, and C. Shahabi. Wsdp [2004]: Web services peer-to-peer discovery service. International Conference on Internet Computing.

[9] Fatih Emekci, Ozgur D. Sahin, Divyakant Agrawal, Amr El Abbadi [2004] : A Peer-to-Peer Framework for Web Service Discovery with Ranking. IEEE International Conference on Web Services.

[10] Gang Zhou, Jianjun Yu, Rui Chen, Hui Zhang [2007]: Scalable Web Service Discovery on P2P Overlay Network. 2007 IEEE International Conference on Services Computing. [11] I. Toma, B. Sapkota, J. Secuila, J. M. Gomez, D. Roman, and C. Bussler [2005]: P2p discovery mechanisms for web service execution environment. Second WSMO Implementation Workshop.

[12] Jacek Kopeck'y [2007]: Semantic Web Service Offer Discovery. OTM 2007 Ws, Part I, LNCS 4805.

[13] Jinghai Raoa, Dimitar Dimitrovb, Paul Hofmannb and Norman Sadeha [2006]: A Mixed Initiative Approach to Semantic Web Service Discovery and Composition :SAP's Guided Procedures Framework.

[14] John Garofalakis, Yannis Panagis, Evangelos Sakkopoulos, Athanasios Tsakalidis [2004] : Web Service Discovery Mechanisms: Looking for a Needle in a Haystack? [15] Jorge Cardoso and Amit Sheth [2002] : Semantic eWorkflow Composition. Technical Report\# 02-004, LSDIS Lab, Computer Science Department, University of Georgia, Athens GA, July 2002.

[16] K. Sivashanmugam, Kunal Verma, Amit Sheth [2004]: Discovery of Web Services in a Federated Registry Environment.

[17] K. Verma, K. Sivashanmugam, Amit Sheth, Abhijit Patil, Swapna Oundhakar, John Miller : METEOR-S WSDI [2003]: A Scalable P2P Infrastructure of Registries for Semantic Publication and Discovery of Web Services.
[18] M. Paolucci, T. Kawamura, T. Payne, and K. Sycara [2002] : Importing the semantic web in UDDI. International Workshop on Web Services, E-Business, and the Semantic Web, pp. 225-236.

[19] M. Paolucci, T. Kawamura, T. Payne, and K. Sycara [2002] Semantic matching of web services capabilities," ISWC, pp. 333-347.

[20] Mario Schlosser, Michael Sintek, Stefan Decker, Wolfgang Nejdl [2002]: A Scalable and Ontology-Based P2P Infrastructure for Semantic Web Services.

[21] Patrick C. K. Hung, Haifei Li [2003]: Web Services Discovery Based on the Trade-off between Quality and Cost of Service: A Token based Approach. ACM SIGecom Exchanges, Vol. 4, No. 2.

[22] Preeda Rajasekaran, John Miller, Kunal Verma, Amit Sheth [2004]: Enhancing Web Services Description and Discovery to Facilitate Composition.

[23] Qiang He, Jun Yan, Yun Yang1, Ryszard Kowalczyk, Hai Jin [2008]: Chord4S: A P2P-based Decentralised Service Discovery Approach. 2008 IEEE International Conference on Services Computing.

[24] Shou-jian Yu, Xiao-kun Ge, Jing-zhou Zhang, Guo-wen Wu [2006]: Web Service Discovery in Large Distributed System Incorporating Semantic Annotations.

[25] Shuiguang Deng, Zhaohui Wu, Jian Wu and Ying Li [2004]: An Efficient Two-Phase Service Discovery Mechanism. WWW 2008, April 2008.

[26] U. Thaden, W. Siberski, and W. Nejdl [2003] : A semantic web Based Peer-to-Peer Service Registry Network, Technical Report, Learning Lab Lower Saxony.

[27] U. Keller, R. Lara, A. Polelres, I. Toma, M. Kifer, and D. Fensel [2004]: WSMO Web Service Discovery. WSMO Working Draft, v0.1.

[28] Ulrich K, Birgitta K, Mirco S., Michael K. DIANE [2007]: An Integrated Approach to Automated Service Discovery, Matchmaking and Composition, International Conference on World Wide Web (WWW'07). 